\title{
Grand challenge: advancing the treatment of neurological disorders by optimizing clinical trials
}

\section{Scott E. Kasner*}

University of Pennsy/vania, Philadelphia, PA, USA

*Correspondence: kasner@mail.med.upenn.edu

Clinical trials provide the best evidence for medical decision-making. The methods for clinical trials are rapidly evolving in response to the needs of every field, and clinical trials involving neurological disorders face unique challenges. Trials are generally launched after many years of painstaking preclinical research and meticulous trial planning, and then require enormous efforts for successful execution. Despite increasing subspecialization of neurology, and especially of neurological research, there are undoubtedly issues that are common to all and represent opportunities for sharing strategies and resources. Frontiers in Clinical Trials in Neurology (FCTN) is a new peer-reviewed, open-access journal launched to provide a broad-based forum to address these critical elements: ethical considerations, study design, measurement of functional status, and methods of analysis across the full spectrum of neurological disorders.

We challenge our colleagues to ask these and many other questions as they apply specifically to clinical trials in neurology:

1. How can we improve recruitment into clinical trials in neurology?

2. How should cognitively impaired patients be enrolled in trials?

3. Who can consent for patients with acute or chronic impairment?
4. Which patients are the most informative for a trial? Are some too mildly or severely affected?

5. How should we measure success in a neurology trial? How should we analyze it?

6. How should we measure disability in a neurology trial? How should we analyze it?

7. Is there a functional outcome measure that can be applied to most neurological disorders?

8. When are non-randomized trials warranted for neurological disorders?

9. What is a clinically meaningful difference in outcomes for sample size considerations?

10. Will the results of a trial be generalizable?

11. Are these questions approached differently by neurologists of various subspecialties?

The results of large randomized clinical trials with prominent positive results will typically be quickly published in the most prominent journals. Neutral and negative trials are frequently published elsewhere and often with substantially longer times until publication. Further, these large trials are usually preceded by decades of smaller pilot, proof-of-concept, surrogate endpoint, and early phase trials, many of which can be hard to find in searchable journal databases if published at all. Detailed protocols of upcoming and ongoing trials also warrant timely publication. FCTN aims to provide a home for trial protocols and results of all types and sizes, with rapid publication and worldwide open access for researchers and clinicians.

We therefore further challenge our colleagues to:

1. Promptly publish protocols for clinical trials in neurology.

2. Report results as soon as possible after trial completion.

3. Fully declare both successes and failures in neurology clinical trials.

FCTN aims to enrich the development and enhance the dissemination of clinical trials in neurological disorders, and to thereby amplify the best evidence for the treatment of our patients.

Received: 06 July 2010; accepted: 19 July 2010; published online: 10 August 2010.

Citation: Kasner SE (2010) Grand challenge: advancing the treatment of neurological disorders by optimizing clinical trials. Front. Neur. 1:17. doi: 10.3389/fneur.2010.00017

This article was submitted to Frontiers in Stroke, a specialty of Frontiers in Neurology

Copyright $\odot 2010$ Kasner. This is an open-access article subject to an exclusive license agreement between the authors and the Frontiers Research Foundation, which permits unrestricted use, distribution, and reproduction in any medium, provided the original authors and source are credited. 